Proceedings of the Institute of Mathematics and Mechanics,

National Academy of Sciences of Azerbaijan

Volume 47, Number 1, 2021, Pages 15-23

https://doi.org/10.30546/2409-4994.47.1.15

\title{
ON STRONG SOLVABILITY OF THE DIRICHLET PROBLEM FOR A CLASS OF SEMILINEAR ELLIPTIC EQUATIONS WITH DISCONTINUOUS COEFFICIENTS
}

\author{
FARMAN I. MAMEDOV AND SHAHLA YU. SALMANOVA
}

\begin{abstract}
We study a strong solvability of the Dirichlet problem

$$
\sum_{i, j=1}^{n} a_{i j}(x) u_{x_{i} x_{j}}+g(x, u)=f(x), x \in \Omega,\left.u\right|_{\partial \Omega}=0
$$

for a class of semilinear elliptic equations with discontinuous coefficients satisfying the Cordes condition. For this problem we get the existence results in $\dot{W}_{2}^{2}(\Omega)$ Sobolev space whenever the norm $\|f\|_{L_{2}(\Omega)}$ is sufficiently small. We also have proved the strong solvability of the Dirichlet problem in space $\dot{W}_{p}^{2}(\Omega)$ with $1<p<\infty$ for the equation considered above with continuous leading coefficients and a small $\|f\|_{L_{p}(\Omega)}$ norm.
\end{abstract}

\section{Introduction and the main results}

Let $E_{n}$ be $n$-dimensional Euclidean space of the points $x=\left(x_{1}, x_{2}, \ldots, x_{n}\right)$ and $\Omega$ be a bounded domain in $E_{n}$ with boundary $\partial \Omega$ of the class $C^{2}$. We consider in $\Omega$ the following Dirichlet problem:

$$
\begin{gathered}
\sum_{i, j=1}^{n} a_{i j}(x) u_{x_{i} x_{j}}+g(x, u)=f(x), x \in \Omega, \\
\left.u\right|_{\partial \Omega}=0 .
\end{gathered}
$$

It is assumed that the coefficients $a_{i j}(x), i, j=1,2, . ., n$ of the operator $L=\sum_{i, j=1}^{n} a_{i j} \frac{\partial^{2}}{\partial x_{i} \partial x_{j}}$ are bounded measurable functions satisfying the conditions

$$
\begin{gathered}
\gamma|\xi|^{2} \leq \sum_{i, j=1}^{n} a_{i j}(x) \xi_{i} \xi_{j} \leq \gamma^{-1}|\xi|^{2}, \forall x \in \Omega, \forall \xi \in E_{n}, \gamma \in(0,1), \\
e s s \sup _{x \in \Omega} \frac{\sum_{i, j=1}^{n} a_{i j}^{2}(x)}{\left[\sum_{i=1}^{n} a_{i i}(x)\right]^{2}} \leq \frac{1}{n-1}-\delta,
\end{gathered}
$$

2000 Mathematics Subject Classification. 35R05, 35J25, 35J60, 35J67, 35R05.

Key words and phrases. semilinear elliptic equations, strong solvability, Dirichlet problem. 
where $\delta \in\left(0, \frac{1}{n}\right)$ is some number and $g(x, u): \Omega \times E_{1} \rightarrow E_{1}$ is a Caratheodory function, measurable with respect to $x \in \Omega$ and, for almost all $x \in \Omega$ continuous with respect to $u \in E_{1}$ and, in addition to this, satisfying the following growth condition:

$$
|g(x, u)| \leq b_{0}|u|^{q}, b_{0}>0 .
$$

Condition (1.4) is called the Cordes condition. We understand it in the following sense: the domain $\Omega$ can be covered by a finite number of domains $\Omega_{i}$ so that in every $\Omega_{i}$ there is a non-degenerate linear transformation of space, under which the equation coefficients satisfy the condition (1.4).

We denote by $\dot{W}_{p}^{2}(\Omega)(p \geq 1)$ the closure of the class of functions $u \in C^{\infty}(\bar{\Omega}) \cap$ $C(\bar{\Omega}),\left.u\right|_{\partial \Omega}=0$ with respect to the norm

$$
\|u\|_{W_{p}^{2}(\Omega)}=\left[\int_{\Omega}\left(|u|^{p}+\sum_{i=1}^{n}\left|u_{i}\right|^{p}+\sum_{i, j=1}^{n}\left|u_{i j}\right|^{p}\right) d x\right]^{1 / p} .
$$

Let $u_{i}$ and $u_{i j}$ denote the derivatives $u_{x_{i}}$ and $u_{x_{i} x_{j}}$ respectively $i ; j=1, \ldots, n$. The conjugate number is denoted by $p^{\prime}$ i.e., $1<p<\infty, \frac{1}{p^{\prime}}+\frac{1}{p}=1$.

The notation $C_{i}(\ldots)$ means that the positive constant $C_{i}$ depends on the parameters indicated in parentheses.

A function $u(x) \in \dot{W}_{p}^{2}(\Omega)$ is called a strong solution of problem (1.1)-(1.2) if it satisfies equation (1.1) a.e. in $\Omega$.

Let us recall some known results directly related to the subject. In the case of linear equations the questions of strong solvability of elliptic and parabolic equations with discontinuous leading coefficients satisfying the Cordes condition were considered in $[3,11,15]$, where the problem of deriving the estimate

$$
\int_{\Omega}(\Delta u)^{2} d x \leq C_{1}(n, \gamma, \delta) \int_{\Omega} L u \cdot \Delta u d x
$$

is the essential point of the investigation. From the examples in [8, p. 48], it follows that if the coefficients of operator $L$ are discontinuous and the Cordes condition is not fulfilled, then the equation $L u=f$ is unsolvable in $\dot{W}_{p}^{2}(\Omega)$ for any $p>1$. For the further results on strong solvability of linear elliptic equations with discontinuous coefficients in $\dot{W}_{p}^{2}(\Omega)(p>1)$ we refer to the monographs $[5,8]$. For such equations with VMO class coefficients the questions of $\dot{W}_{p}^{2}(\Omega)$ strong solvability for $p>1$ were considered in [12]. Studies of nonlinear parabolic equation with discontinuous coefficients were considered in $[3,13,14]$.

Note that, in the study of semilinear equations (1.1) with small nonlinearity $(0<q<1)$, the condition in the norm of a function of type (2.1) on the righthand side is not required (see, [4]).

Different from semilinear equations with regular coefficients, the case of discontinuous coefficients was not considered before on the subject of strong solvability. The aim of this paper is to prove a strong solvability of Dirichlet problem for a class of semilinear elliptic equations (1.1) in Sobolev's space $\dot{W}_{2}^{2}(\Omega)$ when the leading coefficients are discontinuous and satisfy the Cordes condition and the function $f(x)$ has a sufficiently small $L_{2}(\Omega)$ (Cf.[10]) norm. We also consider the results on strong solvability of Dirichlet problem in space $\dot{W}_{p}^{2}(\Omega)$ with $1<p<\infty$ 
for a class of elliptic equations with continuous leading coefficients and function $f(x)$ having a sufficiently small $L_{p}(\Omega)$ norm.

\section{Equations with Discontinuous Coefficients}

Theorem 2.1. Let $n>4,1 \leq q<\frac{n}{n-4}$ and conditions (1.3)-(1.5) be satisfied, $\partial \Omega \in C^{2}$. Then there exists a sufficiently small positive constant $C_{2}=C_{2}\left(n, \gamma, \delta, q, b_{0}\right)$ such that problem (1.1)- (1.2) has at least one solution from $\dot{W}_{2}^{2}(\Omega)$ for any $f(x) \in L_{2}(\Omega)$ satisfying

$$
\|f\|_{L_{2}(\Omega)} \leq C_{2}\left(\operatorname{mes}_{n} \Omega\right)^{\frac{-n+(n-4) q}{2 n(q-1)}} .
$$

Proof. We apply Schauder's theorem on continuous mappings of a convex and the compact set into itself in a Banach space (see, e.g. [5, p. 257]).

Take $L_{2 q}(\Omega)$ as a Banach space. In this space we define the set of functions

$$
V_{2}=\left\{u \in \dot{W}_{2}^{2}(\Omega):\|u\|_{W_{2}^{2}(\Omega)} \leq K\right\},
$$

where the number $K$ will be chosen later. For every $u(x) \in L_{2 q}(\Omega), f(x) \in L_{2}(\Omega)$ we denote by $v(x) \in \dot{W}_{2}^{2}(\Omega)$ the solution of the problem:

$$
\begin{gathered}
L v+g(x, u)=f(x), x \in \Omega, \\
\left.v\right|_{\partial \Omega}=0 .
\end{gathered}
$$

For any $u(x) \in V_{2}, f(x) \in L_{2}(\Omega)$ problem (2.2)-(2.3) is uniquely solvable in the space $\dot{W}_{2}^{2}(\Omega)$, since under our assumptions we come to the Dirichlet problem for the equation

$$
L v=F(x), x \in \Omega,
$$

where $F=f(x)-g(x, u) \in L_{2}(\Omega)($ see $[7])$.

Indeed,

$$
\|F\|_{L_{2}(\Omega)} \leq\|f\|_{L_{2}(\Omega)}+\|g\|_{L_{2}(\Omega)} \leq\|f\|_{L_{2}(\Omega)}+b_{0}\|u\|_{L_{2 q}(\Omega)}^{q} .
$$

$\|u\|_{L_{2 q}(\Omega)}^{q}$ is finite since the space $\dot{W}_{2}^{2}(\Omega)$ is continuously embedded into $L_{2 q}(\Omega)$ for $1 \leq q<\frac{n}{n-4}$, therefore $F \in L_{2}(\Omega)$ [5, see p. 154].

Denote by A the operator which transforms $u$ to $v$ in the problem (2.2), (2.3):

$$
A u=v .
$$

Let us show that the operator $A$ is continuous in $L_{2 q}(\Omega)$.

Let $u_{n} \rightarrow u_{0}$ in $L_{2 q}(\Omega)$ as $n \rightarrow \infty$, where $u_{n}, u_{0} \in L_{2 q}(\Omega)$ and $v_{n}=A u_{n}$; $v_{0}=A u_{0}$. Then

$$
\begin{aligned}
& L v_{n}=-g\left(x, u_{n}\right)+f, \\
& L v_{0}=-g\left(x, u_{0}\right)+f .
\end{aligned}
$$

We show that $v_{n} \rightarrow v_{0}$ in the norm of the space $L_{2 q}(\Omega)$. We have

$$
L\left(v_{n}-v_{0}\right)=-\left(g\left(x, u_{n}\right)-g\left(x, u_{0}\right)\right) .
$$

Having multiplied both parts of equality $(2.5)$ by $\Delta\left(v_{n}-v_{0}\right)$, we obtain:

$$
L\left(v_{n}-v_{0}\right) \Delta\left(v_{n}-v_{0}\right)=-\left(g\left(x, u_{n}\right)-g\left(x, u_{0}\right)\right) \Delta\left(v_{n}-v_{0}\right) .
$$


Hence, using estimate (1.6) and Holder inequality, we obtain

$$
\left\|\Delta\left(v_{n}-v_{0}\right)\right\|_{L_{2}(\Omega)} \leq C_{3}(n, \gamma, \delta)\left\|g_{n}-g\right\|_{L_{2}(\Omega)},
$$

where $g_{n}=g\left(x, u_{n}\right), \quad g=g\left(x, u_{0}\right)$.

Let us show that

$$
\left\|g_{n}-g\right\|_{L_{2}(\Omega)} \rightarrow 0 \text { for } n \rightarrow \infty .
$$

From $u_{m} \rightarrow u$ in $L_{2 q}(\Omega)$ it follows that $u_{m_{k}}-u \rightarrow 0$ almost everywhere in $\Omega$ for some subsequence $\left\{u_{m_{k}}\right\}$. Since $u_{m_{k}} \rightarrow u$ is convergent in $L_{2 q}(\Omega)$, the sequence $\left\{\left|u_{m_{k}}\right|^{2 q}\right\}$ is equi integrable. Therefore the Caratheodory condition and the growth condition (1.5) imply the equicontinuity of the sequence $\left\{\left|g_{m_{k}}-g\right|^{2}\right\}$ and the convergence of $g_{m_{k}}-g \rightarrow 0$ almost everywhere in $\Omega$ as $m_{k} \rightarrow \infty$. Then, applying Vitali's theorem, we obtain

$$
\left\|g_{m_{k}}-g\right\|_{L_{2}(\Omega)} \rightarrow 0 \text { for } m_{k} \rightarrow \infty
$$

The same reasoning also holds for the sequence $\left\{g_{m}-g\right\}$. Indeed, let $\lim _{m_{s} \rightarrow \infty}\left\|g_{m_{s}}-g\right\|_{L_{2}(\Omega)}=\delta, \delta \neq 0$ for some subsequence $\left\{g_{m_{s}}-g\right\}$. Repeating the above arguments for this subsequence, we find that it has a subsequence for which (2.9) is fulfilled.

The obtained contradiction proves the convergence of $\left\|g_{m}-g\right\|_{L_{2}(\Omega)} \rightarrow 0$ as $m \rightarrow \infty$.

Now taking (2.8) into account, from (2.7) we have

$$
\left\|\Delta\left(v_{n}-v_{0}\right)\right\|_{L_{2}(\Omega)} \rightarrow 0,(n \rightarrow \infty) .
$$

Applying the estimate

$$
\left\|v_{n}-v_{0}\right\|_{W_{2}^{2}(\Omega)} \leq C_{3}\left\|\Delta\left(v_{n}-v_{0}\right)\right\|_{L_{2}(\Omega)}
$$

we obtain

$$
\left\|v_{n}-v_{0}\right\|_{W_{2}^{2}(\Omega)} \rightarrow 0 \quad(n \rightarrow \infty) .
$$

By virtue of the embedding theorem $W_{2}^{2}(\Omega) \hookrightarrow L_{2 q}(\Omega)$, we have

$$
\left\|v_{n}-v_{0}\right\|_{L_{2 q}(\Omega)} \rightarrow 0 \text { as } n \rightarrow \infty
$$

The continuity of the operator $A$ has been proved.

Let us prove that the set $V_{2}$ is convex and compact in $L_{2 q}(\Omega)$ and that the operator $A$ transforms it to itself.

For $u_{1}, u_{2} \in V_{2}$ and $v=t u_{1}+(1-t) u_{2}, t \in[0,1]$, we have

$$
\begin{aligned}
& \|v\|_{W_{2}^{2}(\Omega)}=\left\|t u_{1}+(1-t) u_{2}\right\|_{W_{2}^{2}(\Omega)} \leq t\left\|u_{1}\right\|_{W_{2}^{2}(\Omega)}+ \\
& +(1-t)\left\|u_{2}\right\|_{W_{2}^{2}(\Omega)} \leq t K+(1-t) K=K,
\end{aligned}
$$

which means the convexity of $V_{2}$.

The set $V_{2} \subset L_{2 q}(\Omega)$ is compact by virtue of the theorem on compact embed$\operatorname{ding} W_{2}^{2} \hookrightarrow L_{2 q}(\Omega)$ whenever

$$
1 \leq q<\frac{n}{n-4}
$$

Let us show that for a certain choice of $K$ the operator $A$ transforms $V_{2}$ to itself. 
For the solution of the Dirichlet problem for equation (2.4) we have

$$
\|v\|_{W_{2}^{2}(\Omega)} \leq C_{4}(n, \gamma, \delta)\|F\|_{L_{2}(\Omega)} \leq C_{4}\left[\|f\|_{L_{2}(\Omega)}+\|g\|_{L_{2}(\Omega)}\right] .
$$

Furthermore,

$$
\begin{gathered}
\|g\|_{L_{2}(\Omega)} \leq\left(\int_{\Omega} b_{0}^{2}|u|^{2 q} d x\right)^{1 / 2} \leq b_{0}\left(m e s_{n} \Omega\right)^{\frac{1}{2}-\frac{n-4}{n} q}\left(\int_{\Omega} u^{\frac{2 n}{n-4}} d x\right)^{\frac{n-4}{2 n} q} \leq \\
\leq b_{0}\left(\operatorname{mes}_{n} \Omega\right)^{\frac{1}{2}\left(1-\frac{n-4}{n} q\right)} C_{5}(n, q)\|u\|_{W_{2}^{2}(\Omega)}^{q} .
\end{gathered}
$$

Here we have used the above-mentioned embedding theorem for (2.10). Using the estimate (2.12) in (2.11), we obtain

$$
\begin{aligned}
\|v\|_{W_{2}^{2}(\Omega)} & \leq C_{6}\left[b_{0}\left(\text { mes }_{n} \Omega\right)^{\frac{1}{2}\left(1-\frac{n-4}{n} q\right)}\|u\|_{W_{2}^{2}(\Omega)}^{q}+\|f\|_{L_{2}(\Omega)}\right] \leq \\
& \leq C_{6}\left[K^{q} b_{0}\left(\text { mes }_{n} \Omega\right)^{\frac{1}{2}\left(1-\frac{n-4}{n} q\right)}+\|f\|_{L_{2}(\Omega)}\right],
\end{aligned}
$$

where $C_{6}=C_{6}(n, \delta, \gamma, q)$.

Let us assume that $K$ satisfies the following estimate

$$
\left[K^{q} b_{0}\left(m e s_{n} \Omega\right)^{\frac{1}{2}\left(1-\frac{n-4}{n} q\right)}+\|f\|_{L_{2}(\Omega)}\right] \leq K .
$$

For such a number $K$ to exist it is sufficient that

$$
\|f\|_{L_{2}(\Omega)} \leq C_{7}\left(\operatorname{mes}_{n} \Omega\right)^{-\left(\frac{n-(n-4) q}{2 n(q-1)}\right)}
$$

where $C_{7}=C_{7}(n, \delta, \gamma, q)$.

Indeed, if we introduce the notation

$$
a=b_{0}\left(m e s_{n} \Omega\right)^{\frac{1}{2}\left(1-\frac{n-4}{n} q\right)}, \quad b=\|f\|_{L_{2}(\Omega)},
$$

then inequality $(2.13)$ reads

$$
a K^{q}+b \leq K, a K^{q}-K+b \leq 0, \quad K>0 .
$$

The function $\Psi(K)=a K^{q}-K, K \geq 0$, takes a minimal value for $K_{0}=$ $\left(\frac{1}{q a}\right)^{\frac{1}{q-1}}$. Indeed, $\Psi^{\prime}(K)=a q K^{q-1}-1$, then for $K_{0}^{q-1}=\frac{1}{q a}$ we have $\Psi^{\prime}\left(K_{0}\right)=0$, $\Psi^{\prime \prime}\left(K_{0}\right)>0$. Therefore, for $b \leq \Psi\left(K_{0}\right)$ inequality (2.13) is solvable with respect to $K$. Theorem 2.1 is proved.

In the case $1 \leq n \leq 4$ the following statement is true.

Theorem 2.2. Let conditions (1.3)-(1.5) be fulfilled and $1 \leq n<4(n=4), 1<$ $q<\infty, \partial \Omega \in C^{2}$. Then there exists a sufficiently small positive constant $C_{8}=$ $C_{8}\left(n, \gamma, \delta, q, b_{0}\right)\left(C_{9}=C_{9}\left(n, \gamma, \delta, q, b_{0}\right)\right)$ such that problem (1.1), (1.2) has at least one solution from $\dot{W}_{2}^{2}(\Omega)$ for any $f(x) \in L_{2}(\Omega)$ satisfying the condition

$$
\|f\|_{L_{2}(\Omega)} \leq C_{8}\left(m e s_{n} \Omega\right)^{-\frac{n+q(n-4)}{2 n(q-1)}} \quad\left(\|f\|_{L_{2}(\Omega)} \leq C_{9}\left(m e s_{n} \Omega\right)^{-\frac{1}{2(q-1)}}\right) .
$$

In proving this theorem, as a Banach space we take the space $C(\bar{\Omega})\left(L_{2 q}(\Omega)\right)$ and argue as above applying the theorem of compact embedding $W_{2}^{2}(\Omega) \hookrightarrow C(\bar{\Omega})$ $\left(W_{2}^{2}(\Omega) \hookrightarrow L_{2 q}(\Omega)\right)$. 


\section{Equations with Continuous Coefficients}

In this section, we will consider the Dirichlet problem for semilinear elliptic equations (1.1) with continuous leading coefficients. In other words, we will consider, in $\Omega$, the Dirichlet problem

$$
\begin{gathered}
\sum_{i, j=1}^{n} a_{i j}(x) u_{i j}+g(x, u)=f(x), \quad x \in \Omega, \\
\left.u\right|_{\partial \Omega}=0,
\end{gathered}
$$

when the coefficients $a_{i j}(x), i, j=1,2, \ldots, n$ of the operator $L=\sum_{i, j=1}^{n} a_{i j}(x) \frac{\partial^{2}}{\partial x_{i} \partial x_{j}}$ are functions satisfying conditions (1.3) such that

$$
a_{i j}(x) \in C(\bar{\Omega}), i, j=1,2 \ldots, n .
$$

Note that in the preceding section our consideration was confined to the case $1 \leq q<\frac{n}{n-4}, n>4$. This was caused by the application of existing a priori estimate (1.6) to an equation with discontinuous coefficients.

In the case of equations with continuous coefficients we will apply a priori estimate in $W_{p}^{2}(\Omega)$ ( see, e.g. [5, Lemma 9.17]).

The following statement is true.

Theorem 3.1. Let conditions (1.3), (1.5) and (3.3) be fulfilled, $1<q<\infty$, $p>\frac{n}{2 q^{\prime}}, p \geq 1, \partial \Omega \in C^{2}$. Then there exists a sufficiently small positive constant $C_{10}=C_{10}\left(n, \gamma, \delta, q, b_{0}\right)$ such that problem (3.1), (3.2) has at least one solution from $\dot{W}_{p}^{2}(\Omega)$ for any $f(x) \in L_{p}(\Omega)$ satisfying

$$
\|f\|_{L_{p}(\Omega)} \leq C_{10}\left(\operatorname{mes}_{n} \Omega\right)^{-\frac{n+(n-2 p) q}{p n(q-1)}} .
$$

Proof. In this case, as a Banach space take: $L_{p q}(\Omega)$ if $\frac{n}{2 q^{\prime}}<p<\frac{n}{2}$, $p \geq 1 ; C^{\alpha}(\Omega) \quad\left(0<\alpha<2-\frac{n}{p}\right)$ if $\frac{n}{2}<p \leq n ; C^{1, \alpha}(\Omega) \quad\left(0<\alpha<1-\frac{n}{p}\right)$ if $p>n$.

Assume $V_{p}=\left\{u \in \dot{W}_{p}^{2}(\Omega):\|u\|_{W_{p}^{2}(\Omega)} \leq K\right\}$.

Denote by $v(x) \in \dot{W}_{p}^{2}(\Omega)$ the solution of the problem

$$
\begin{gathered}
L v+g(x, u)=f(x), \quad x \in \Omega, \\
\left.v\right|_{\partial \Omega}=0,
\end{gathered}
$$

where $u(x)$ is an arbitrary function from $L_{p q}$ if $\frac{n}{2 q^{\prime}}<p<\frac{n}{2}, p \geq 1 ; C^{\alpha}(\Omega)$ $\left(0<\alpha<2-\frac{n}{p}\right)$ if $\frac{n}{2}<p \leq n ; C^{1, \alpha}(\Omega)\left(0<\alpha<1-\frac{n}{p}\right)$ if $p>n$.

For any $u(x) \in V_{p}, f \in L_{p}(\Omega)$ problem (3.5)-(3.6) is solvable in the spaces $\dot{W}_{p}^{2}(\Omega)$ since under these assumptions we deal with the solvability of the Dirichlet problem for equation (2.4), where $F=f(x)-g(x, u) \in L_{p}(\Omega)$ (for the solvability of the Dirichlet problem [5, Theorem 9.15]).

By virtue of the embedding $W_{p}^{2}(\Omega) \hookrightarrow L_{p q}(\Omega)$ we have

$$
\|F\|_{L_{p}(\Omega)} \leq\|f\|_{L_{p}(\Omega)}+\|g\|_{L_{p}(\Omega)} \leq\|f\|_{L_{p}(\Omega)}+b_{0}\|u\|_{L_{p q}(\Omega)}^{q},
$$


therefore $F \in L_{p}(\Omega)$.

Denote by $A$ the operator transforming $u$ to $v$ and show that the operator $A$ is continuous. For that, let $u_{n} \rightarrow u_{0}$ in $L_{p q}(\Omega)$ as $n \rightarrow \infty, u_{n}, u_{0} \in L_{p q}(\Omega)$ and $v_{n}, v_{0}$ be solutions of $(2.4)$ in $W_{p}^{2}(\Omega)$. Then $v_{n}-v_{0}$ satisfies (2.5). By virtue of the priori estimate

$$
\|v\|_{W_{p}^{2}(\Omega)} \leq C_{11}(n, \gamma, p)\|L v\|_{L_{p}(\Omega)}, \quad v \in W_{p}^{2}(\Omega)
$$

it follows

$$
\left\|v_{n}-v_{0}\right\|_{W_{p}^{2}(\Omega)} \leq C_{11}\left\|g_{n}-g\right\|_{L_{p}(\Omega)} .
$$

Repeating the reasoning of the proof of Theorem 2.1 we get

$$
\left\|v_{n}-v_{0}\right\|_{W_{p}^{2}(\Omega)} \rightarrow 0(n \rightarrow \infty) .
$$

This implies

$$
\left\|v_{n}-v_{0}\right\|_{L_{p q}(\Omega)} \rightarrow 0 \text { as } n \rightarrow \infty .
$$

The compactness of $V_{p}$ follows from the compact embedding $W_{p}^{2}(\Omega)$ into $L_{p q}(\Omega)$, while the convexity is obvious.

Let us show that for a certain choice of $K$ the operator $A$ transforms $V_{p}$ to itself. For the solution of the Dirichlet problem (3.1) we have the estimate

$$
\begin{gathered}
\|v\|_{W_{p}^{2}(\Omega)} \leq C_{12}(\gamma, n)\|L v\|_{L_{p}(\Omega)} \leq C_{12}\left[\|g\|_{L_{p}(\Omega)}+\|f\|_{L_{p}(\Omega)}\right] \leq \\
\leq C_{13}\left[\left(m e s_{n} \Omega\right)^{\frac{1}{p}\left(1-\frac{n-2 p}{n} q\right)} b_{0}\|u\|_{W_{p}^{2}(\Omega)}^{q}+\|f\|_{L_{p}(\Omega)}\right] \leq \\
\leq C_{13}\left[\left(\text { mes }_{n} \Omega\right)^{\frac{1}{p}\left(1-\frac{n-2 p}{n} q\right)} b_{0} K^{q}+\|f\|_{L_{p}(\Omega)}\right],
\end{gathered}
$$

where $C_{13}=C_{13}\left(n, \gamma, \delta, q, b_{0}\right)$.

From (3.4) it follows that, the inequality

$$
C_{13}\left[K^{q} b_{0}\left(m e s_{n} \Omega\right)^{\frac{1}{2}\left(1-\frac{n-2 p}{n} q\right)}+\|f\|_{L_{p}(\Omega)}\right] \leq K
$$

is solvable with respect to $K>0$, i.e., the operator $A$ transforms $V_{p}$ to itself.

Theorem 3.1 has been proved.

Remark 3.1. The assertion of Theorem 3.1 remains valid in the case of nonlinear equation

$$
\sum_{i, j=1}^{n} a_{i j}(x)\left(x, u, u_{x}\right) u_{x_{i} x_{j}}+g(x, u)=f(x)
$$

with the Cordes type condition

$$
\underset{x \in \Omega}{\operatorname{essup}} \frac{\sum_{i, j=1}^{n} a_{i j}^{2}(x, \xi, \eta)}{\left(\sum_{i=1}^{n} a_{i i}^{2}(x, \xi, \eta)\right)^{2}} \leq \frac{1}{n-1}-\delta ; \quad \xi \in E_{1}, \quad \eta \in E_{n} .
$$

As to the existence of positive solutions of problem (3.1), (3.2) we make the following

Remark 3.2. Let all the conditions of Theorem 3.1 be fulfilled for the operator $L$, domain $\Omega$ and positive functions $g(x, u)$ and $f(x)$. Let further $p \geq n$. Then 
there exists a sufficiently small positive constant $C_{14}=C_{14}\left(\gamma, n, p, q, b_{0}\right)$ such that problem (3.1), (3.2) has at least one positive solution from $\dot{W}_{p}^{2}(\Omega)$ for any $f(x) \in L_{p}(\Omega)$ satisfying the condition

$$
\|f\|_{L_{p}(\Omega)} \leq C_{14}\left(m_{e} s_{n} \Omega\right)^{-\frac{n+(n-2 p) q}{p n(q-1)}} .
$$

The proof of this statement is based on that of Theorem 3.1. In this case the class $V_{p}$ is defined in the form

$$
V_{p}=\left\{u \in \dot{W}_{p}^{2}(\Omega):\|v\|_{W_{p}^{2}(\Omega)} \leq K, \quad u(x)>0, x \in \Omega\right\} .
$$

The class $V_{p}$ is preserved by the operator $A$ if we use the reasoning of Theorem 3.1 and the maximum principle [2] for solutions of elliptic equations in the space $W_{p}^{2}(\Omega)$ for $p \leq n$.

\section{References}

[1] D.R.Adams and M.Pierre, Capacitary strong type estimates in semilinear problems. Ann. Inst.Fourier (Grenoble), 41 (1) (1991), 117-135.

[2] A.D.Alexandrov, Investigations on the maximum principle.Izv.Vyssh.Uchebn. Zaved.Mat., 1(20) (1961), 3-20.

[3] Yu.A.Alkhutov and I.T.Mamedov, The first boundary value problem for nondivergent parabolic equations of second order with discontinuous coefficients. Matem.Sb. (N. S.), 131(173) (4(12)) (1986), 477-500. (in Russian)

[4] P.Dravek, A.Kufner, F.Nicolosi, Nonlinear elliptic equations: Singular and Degenerate Case. University of West Bohemia, Pilsen, 1996.

[5] D.Gilbarg and N.Trudinger, Elliptic differential equations with partial derivatives of second order. Nauka, Moscow, 1989.

[6] N.J.Kalton and J.E.Verbitsky, Nonlinear equations and weighted norm inequalities. Trans. Amer. Math. Soc., 351(9) (1999), 3441-3497.

[7] O.A.Ladizhenskaya, The simple proof of solvability of basic boundary value problem and of problem on egihenvalues for linear equations. Vestnik $L G U, \mathbf{1 1}(11)$ (1955), 23-29. (in Russian)

[8] O.A.Ladizhenskaya and N.N.Uraltseva, Linear and quasilinear equations of elliptic type. Nauka, Moscow, 1973. (in Russian)

[9] P.L.Lions, On the existence of positive solutions ofsemilinear elliptic equations. SIAM Review, 24(4) (1982), 441-467.

[10] F.I.Mamedov and Sh.Yu.Salmanova, On strong solvability of the dirichlet problem for semilinear elliptic equations with discontinuous coefficients. Trans.Natl. Acad. Sci. Azerb. Ser. Phys.-Tech. Math. Sci., 27(7)(2007), 117-124.

[11] G.Talenti, Sopra una classe di equazione ellitiche a cofficienti misurableili. Ann. Mat.Pura Appl., 69(1) (1965), 285-304.

[12] C.Vitanza, A new contribution to the $W_{p}^{2}$ regularity for a class of elliptic second order equations with discontinuous coefficients. Le matematiche, 48(2)(1993), 287296.

[13] G.C.Wen, Initial-mixed boundary value problems for parabolic equations of second order with measurable coefficients in a higher dimensional domain. Proceedings of the second ISAAC Congress, 1 (2000), 185-192.

[14] G.C.Wen, TainMaoying, Initial-oblique derivative problems for nonlinear parabolic equations with measurable coefficients.Comm. in Nonlinear Sci. and Numer.Simu., 3(2) (1998), 109-113. 
[15] V.V.Zhikov and M.M.Sirazudinov, On the $G$-compactness of a class of nondivergence elliptic operators of second order.Math. USSR-Izv., 19(1) (1982), 27-40.

Farman I. Mamedov

Oil and Gas Scientific Research Project Institute of SOCAR Company., Baku, Azerbaijan

E-mail address: farman-m@mail.ru

Shahla Yu. Salmanova

Institute Mathematics and Mechanics of NAS of Azerbaijan, Baku, Azerbaijan E-mail address: shehla_shukurova@mail.ru

Received: May 12, 2020; Revised: November 3, 2020; Accepted: November 23, 2020 